\title{
Pankreaskarzinomchirurgie Wie groß ist das Optimierungspotenzial?
}

\author{
Hintergrund und Fragestellung \\ Je nach Krankenhaus unterscheiden sich die Kom- \\ plikationsraten nach Pankreaskarzinomchirurgie \\ erheblich. Zum gegenwärtigen Zeitpunkt existie- \\ ren keine gültigen Qualitätsindikatoren, die eine \\ Beurteilung des Managements einer Pankreaskar- \\ zinombehandlung erlauben.
}

\section{Patienten und Methodik}

43 gültige Qualitätsindikatoren wurden aus der Literatur, anhand von Konsensuskonferenzen und in Expertenbefragungen identifiziert und von einem aus 20 Experten bestehenden Panel bewertet.

Anhand von Pankreaskarzinomdaten des amerikanischen nationalen Krebsregisters (49.065 Patienten und 1.134 Krankenhäuser) wurde die Einhaltung der identifizierten Indikatoren in Bezug auf den Patienten und das Krankenhaus untersucht.

\section{Ergebnisse}

Die 43 identifizierten Qualitätsindikatoren beinhalteten folgende Faktoren: in elf Punkten die Behandlungsstruktur (25\%), in 19 Punkten klinische
Behandlungsprozesse (44\%), in vier Punkten die Behandlungseffizienz (9\%) und in fünf Punkten das Patientenoutcome (12\%). Die Einhaltung der identifizierten Qualitätsindikatoren variierte zwischen den Krankenhäusern bezogen auf die untersuchten Patienten $(\mathrm{n}=49065)$ von 50-97\%, bezogen auf die untersuchten Krankenhäuser ( $\mathrm{n}=$ 1.134) von $7-100 \%$. Zehn der 43 Schlüsselindikatoren für Behandlungsqualität wurden von weniger als $50 \%$ der Krankenhäuser erfüllt.

\section{Schlussfolgerungen}

Basierend auf den identifizierten Qualitätsindikatoren besteht eine erhebliche Variabilität in der Qualität der Pankreaskarzinombehandlung in den USA. Die Anwendung der identifizierten Qualitätsindikatoren ermöglicht Krankenhäusern die eigene Beurteilung sowie die Optimierung ihrer Pankreaskarzinombehandlung.

Schlüsselwörter: Pankreaskarzinomchirurgie Pankreaskarzinombehandlung - Qualitätsindikatoren - Optimierungspotenzial
Bilimoria KY et al. Assessment of pancreatic cancer care in the United States based on formally developed quality indicators.

J Natl Cancer Inst 2009;101:848-59.

\section{Mindestmengen und Qualitätssicherung bei OP unabdingbar Kommentar von Jörg Kleef}

D ie Pankreaskarzinomchirurgie hat sich in den letzten Jahrzehnten vor allem in spezialisierten Zentren weiterentwickelt. Dies konnte durch eine zunehmende Erfahrung, neue chirurgische Techniken, interdisziplinäre Behandlungskonzepte und eine verbesserte perioperative Versorgung erreicht werden.

Für keine andere Operation ist der Zusammenhang von Fallzahl mit den postoperativen Ergebnissen ähnlich gut belegt wie für Pankreasresektionen. Dies zeigt sich in großen epidemiologischen Studien und in Metaanalysen und gilt sowohl für die perioperative Mortalität als auch für das langfristige Überleben. Die Konsequenzen, die sich aus dieser Erkenntnis ergeben müssten, werden sowohl in den Vereinigten Staaten als auch sicherlich in Deutschland nicht konsequent umgesetzt. Dies zeigen erneut die vorliegenden Daten.
Ein Qualitätsindikator, nämlich die Fallzahl, die bei zwölf oder mehr Pankreasresektionen pro Jahr festgesetzt wurde, wurde nur von knapp 7\% der über 1000 untersuchten Krankenhäuser eingehalten, das heißt die größte Anzahl der Pankreasresektionen finden in Kliniken statt, die eben nicht die notwendige Erfahrung mit dieser Operationstechnik aufweisen können. Ähnliche Daten könnten wohl sicherlich auch in Deutschland erhoben werden.

Die anderen von den Autoren der Untersuchung vorgeschlagenen Qualitätsindikatoren erscheinen zum Teil trivial wie beispielsweise die Forderung, dass wenn Krankenhäuser Pankreasresektionen durchführen, das Tumor-Stadium, die Histologie, das Grading und der Resektionsstatus sowie die untersuchten Lymphknoten dokumentiert werden, sowie beispielsweise, dass Patienten

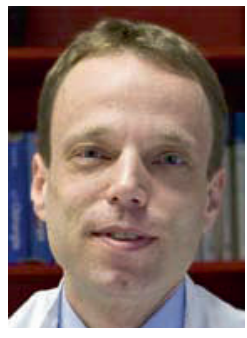

PD Dr. med. Jörg Kleeff Klinikum rechts der Isar, München E-Mail: joerg.kleeff@ chir.med.tu-muenchen.de 